\title{
Sound pulse broadening in stressed granular media
}

\author{
Vincent Langlois* \\ Université Paris-Est, Laboratoire Géomatériaux et Environnement (EA 4508), UPEM, F-77454 Marne-la-Vallée, France \\ Xiaoping $\mathrm{Jia}^{\dagger}$ \\ Institut Langevin, ESPCI ParisTech, CNRS UMR 7587, 1 rue Jussieu, 75005 Paris, France \\ and Université Paris-Est, 5 bd Descartes, 77454 Marne-la-Vallée Cedex 2, France
}

(Received 14 November 2014; published 17 February 2015)

\begin{abstract}
The pulse broadening and decay of coherent sound waves propagating in disordered granular media are investigated. We find that the pulse width of these compressional waves is broadened when the disorder is increased by mixing the beads made of different materials. To identify the responsible mechanism for the pulse broadening, we also perform the acoustic attenuation measurement by spectral analysis and the numerical simulation of pulsed sound wave propagation along one-dimensional disordered elastic chains. The qualitative agreement between experiment and simulation reveals a dominant mechanism by scattering attenuation at the high-frequency range, which is consistent with theoretical models of sound wave scattering in strongly random media via a correlation length.
\end{abstract}

DOI: 10.1103/PhysRevE.91.022205

PACS number(s): 45.70. $-\mathrm{n}, 43.35 .+\mathrm{d}, 81.05 . \mathrm{Rm}$

\section{INTRODUCTION}

The structure of jammed granular media is characterized by the inhomogeneous contact networks at the mesoscopic scale [1]. Elastic waves in such media propagate in different ways according to the ratio of the wavelength $\lambda$ to the grain size $d$. At low $\lambda / d$ the propagation of waves is incoherent and strongly scattered, while at high $\lambda / d$ the wave propagation is coherent and ballistic [2]. The scattered waves are the fingerprint of a specific configuration of the contact force network, which can be used to measure the interfacial dissipation on the grain scale and detect the tiny rearrangement of the contact network [3]. On the other hand, the velocity measurement of coherent waves allows one to characterize the elastic properties of granular packings on the macroscopic scale and infer the coordination number within the framework of effective medium theories [4-7]. This latter plays a central role in the granular mechanics [8-11].

Pulse wave propagation provides a convenient way for material characterization and signal transmission, thanks to the possible temporal separation of signals from parasites. In disordered granular packings, both experiments and numerical simulations have shown that coherent wave pulses, compressional or shear, decay in amplitude and broaden in width as they propagate, thus reducing significantly the resolution capacity [2,12-16]. In general, two mechanisms may be responsible for the pulse broadening, i.e., attenuation and dispersion of velocity, which can be analyzed in the frequency domain via the dispersion relationship $k(\omega)$ between the wave number $k$ and the angular frequency $\omega$. Indeed, from $k(\omega)=k^{\prime}(\omega)+i k^{\prime \prime}(\omega)$, the phase velocity $V(\omega)=\omega / k^{\prime}$ and the attenuation $\alpha(\omega)=k^{\prime \prime}$ can be deduced, respectively. Somfai et al. [14] showed that in (one-dimensional) ordered granular media, the dispersion effect due to discrete lattice governs the shape of the coherent wave front, and leads to

\footnotetext{
*vincent.langlois@u-pem.fr

${ }^{\dagger}$ xiaoping.jia@espci.fr
}

a pulse broadening which scales with the source-detector distance $L$ as $\propto L^{1 / 3}$. By introducing the polydispersity in the one-dimensional (1D) chain, their numerical simulations revealed that disorder increases the pulse width. It was also shown in amorphous media that disorder could induce a dispersion of the phase velocity particularly at the highfrequency range with $\lambda \sim d$ [17]. However, at such frequency range, the coherent waves often exhibit strong attenuation dominated by strong scattering thus being responsible for the pulse broadening $[18,19]$. In granular media, despite some recent numerical studies [14-16], few experimental data are actually available to highlight the interplay between pulse broadening and attenuation.

In this work, we study the propagation of compressional wave pulses through disordered granular packings under stress. A particular attention is focused on the evolution of the pulse width of the coherent sound wave as a function of propagation distance. Various granular media are investigated, including the mixture of glass and (poly)methyl-methacrylate (PMMA) beads with enhanced disorder. To understand the origin of the pulse broadening, we also perform the attenuation measurement by a spectral analysis and numerical simulations of the pulse propagation along disordered 1D elastic chains. The existing models of elastic wave scattering in random media are finally discussed to highlight the attenuation mechanism in granular media.

\section{EXPERIMENTS}

\section{A. Granular samples}

Acoustic measurements are performed using a pulse transmission through granular media under stress [2]. The beads are poured by rain deposition into a rigid cell of inner diameter $30 \mathrm{~mm}$. A plane-wave source (longitudinal) transducer of diameter $30 \mathrm{~mm}$ is placed on the top of the cell and the wave transmission is detected by a similar transducer on the bottom; both the source and the detector are in direct contact with the beads (Fig. 1). Before the ultrasonic measurements, two 


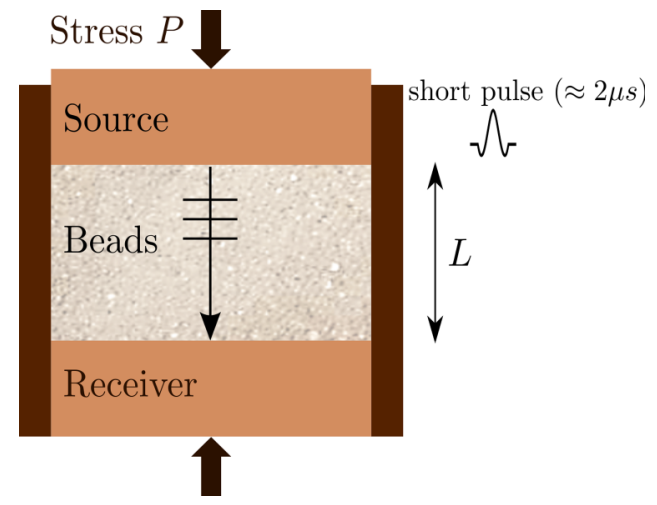

FIG. 1. (Color online) Sketch of the experimental setup.

cycles of loading and unloading are performed to improve the reproducibility of measurements.

Various granular samples are investigated in this study. Table I summarizes the main characteristics like constitutive material (glass, PMMA, or steel), mean size of beads, polydispersity, and sphericity. The beads with a mean diameter larger than $1 \mathrm{~mm}$ have a good spherical shape and a low polydispersity, while the smaller ones present sometimes a nonspherical shape (e.g., partial coalescence of two beads) and higher polydispersity. The use of polymeric beads, e.g., PMMA, allows us to evaluate the effect of dissipation mechanisms on the coherent wave front. Indeed, by measuring the absorption time (not shown here) from the time-resolved intensity profile of scattered waves [20,21], we infer a wave dissipation four times larger in PMMA beads than in glass beads at $300 \mathrm{kHz}$, due to the viscoelastic loss.

\section{B. Impulse response and wave velocity measurement}

To obtain the impulse response of the coherent compressional wave in granular media, we may deconvolve the signals transmitted through the media by the signal $S$ sent by the source transducer. Figure 2 shows an example of the measured signal and deconvolved one (first part). The source signal $S$ [inset of Fig. 2(b)] is obtained by putting the source against the detector via a coupling film. The deconvolution calculation, based on FFT, is performed on the frequency range [1-900 kHz]. As in [14], we characterize the impulse response of the coherent signal by the arrival times associated to three particular points: the peak $A_{1}\left(t_{1}\right)$, the first arrival at $10 \%$ of the peak $\left(t_{0}\right)$, and the first zero crossing $\left(t_{2}\right)$. Complementary measurements of pulse transmission were also performed in a reference medium, i.e., water filling up the above cell (waterproof) in which the shape of received signal does not evolve significantly as the distance source-receiver increases. This observation indicates that the
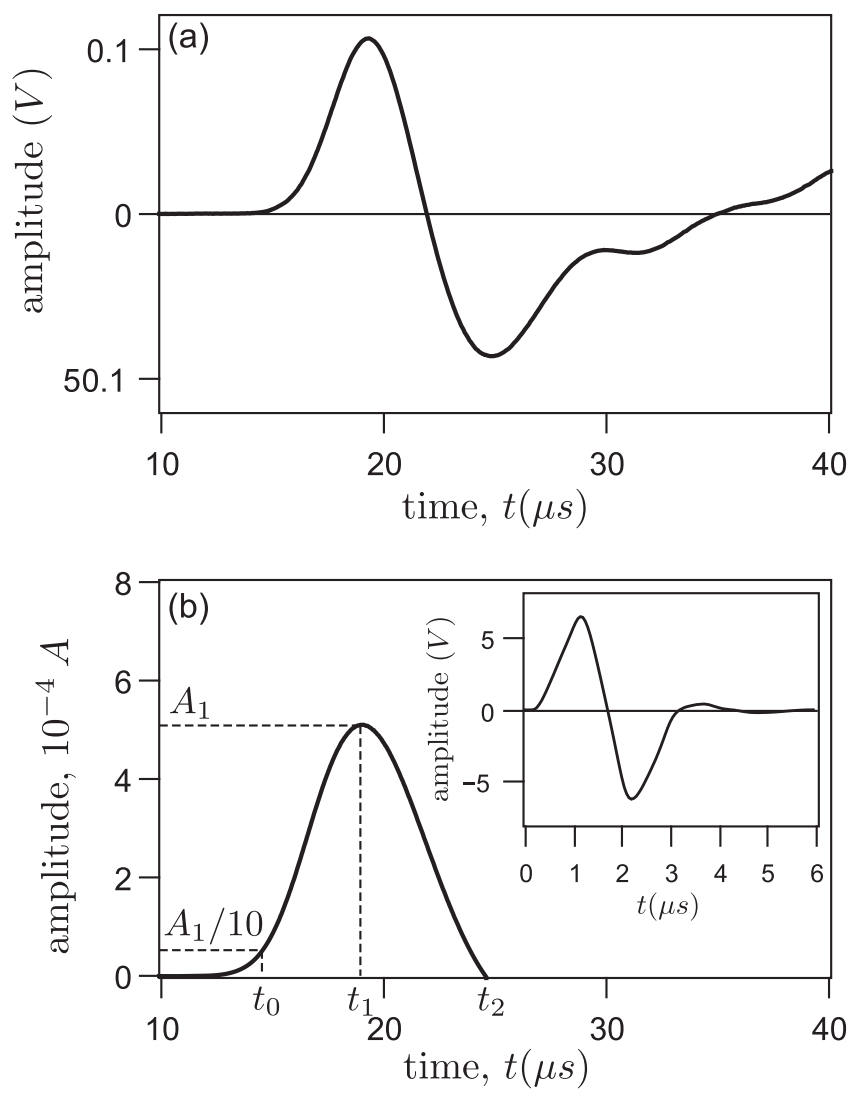

FIG. 2. (a) Measured pulse of a compressional wave measured in a glass bead packing of $d=0.715 \mathrm{~mm}$, under stress $P=995 \mathrm{kPa}$, and at a distance $L=21.7 \mathrm{~mm}$. (b) The deconvolved pulse of the transmitted signal (first part), by a reference signal from the source traducer (inset).

source transducer does generate a quasiplane wave and the effect of edge waves are negligible in our experiments [22].

In pulse transmission experiments, the wave velocity may be determined via the time-of-flight method, making measurements at two different distances. If only one-distance measurement is conducted for the velocity calculation, the different time $t_{0}, t_{1}$, or $t_{2}$ leads obviously to different values (Fig. 3). Notice however that the velocity determined with $t_{1}$ is almost independent of the source-receiver distance, contrary to the other times used. As shown in Fig. 3, this velocity determined under low stress may exhibit a slight increase with the distance source receiver. Such discrepancy could be improved by an alternative method based on the Hilbert transform using a reference signal, for example, the source signal [23]. Nevertheless, the difference between the velocity measured via $t_{1}$ and that determined by the alternative method is no more than $5 \%$.

TABLE I. Description of the beads used for granular packings.

\begin{tabular}{|c|c|c|c|c|c|c|c|c|c|c|c|}
\hline Material & & & & & & Glass & & & & Steel & PMMA \\
\hline $\begin{array}{l}\text { Size } d(\mathrm{~mm}) \\
\text { Tolerance }(\mathrm{mm}) \\
\text { Sphericity }\end{array}$ & 5 & 4 & $\begin{array}{c}3 \\
\pm 0.01 \\
\text { good }\end{array}$ & 2 & 1.5 & $\begin{array}{c}1.125 \\
\pm 0.125 \\
\text { quite good }\end{array}$ & $\begin{array}{c}0.715 \\
\pm 0.085 \\
\text { quite good }\end{array}$ & $\begin{array}{c}0.35 \\
\pm 0.05 \\
\text { poor }\end{array}$ & $\begin{array}{c}0.225 \\
\pm 0.025 \\
\text { poor }\end{array}$ & $\begin{array}{c}0.7 \\
\pm 0.1 \\
\text { poor }\end{array}$ & $\begin{array}{c}0.615 \\
\pm 0.015 \\
\text { quite good }\end{array}$ \\
\hline
\end{tabular}




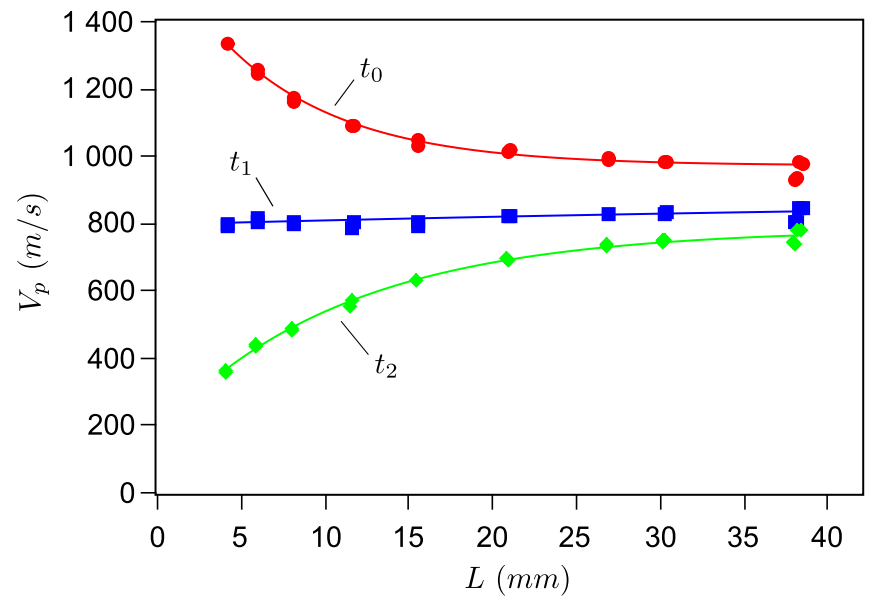

FIG. 3. (Color online) Compressional wave velocities measured as a function of propagation distance, using different time arrivals (see text).

The wave velocity measurement has been extensively used to study the nonlinear elasticity of stressed granular media; see, e.g., $[4,6]$. Figure $4(a)$ shows that as the confining pressure is decreased, there is an increase of the time of flight, a decay of the pulse amplitude, and a broadening of the pulse. However, by rescaling the time $t / t_{1}$ and the amplitude $A / A_{1}$ axes, we find that almost all responses collapse [Fig. 4(b)], indicating that the coherent wave fronts are mainly governed by the time scale $t / t_{1}$ or $V_{p} t$. To evaluate the accuracy of this collapse, we compare the normalized width of the signal, $W=\left(t_{1}-t_{0}\right) / t_{1}$ at high and low pressures (inset of Fig. 4). The normalized width at low pressure is always higher than that obtained at high pressure. This difference may arise from the heterogeneity of the contact force networks which is more important at lower confining pressure [24]. For glass bead packings, the collapse of the rescaled responses appears more effective with beads of large size, less polydisperse, and more spherical whose packing structures are expected to be less disordered. These results would suggest a possible dependence of the normalized width on the amount of disorder.

\section{Evolution of sound pulse width}

Figure 5(a) shows a pulse broadening and decay with the distance of propagation through granular media. The width of the signal, $t_{1}-t_{0}$, scales with the ratio $L / d$ as a power law with exponent of about $1 / 2$ or higher for beads of large size [Fig. 5(b)]. However, if the pulse width is normalized by the propagation time $t_{1}$, it decreases with the propagation distance $L / d$, according to

$$
W \simeq C_{W}(L / d)^{-0.5},
$$

where $C_{W}$ is a prefactor. Moreover, Fig. 5(c) shows that the data of these normalized widths are much less disperse compared to those in Fig. 5(b), ranged in a grey band likely independent of the material property of beads and its mean size $d$. The data located in the upper part of this band are obtained with less spherical small beads. The results obtained from PMMA beads are very similar to those from glass beads,
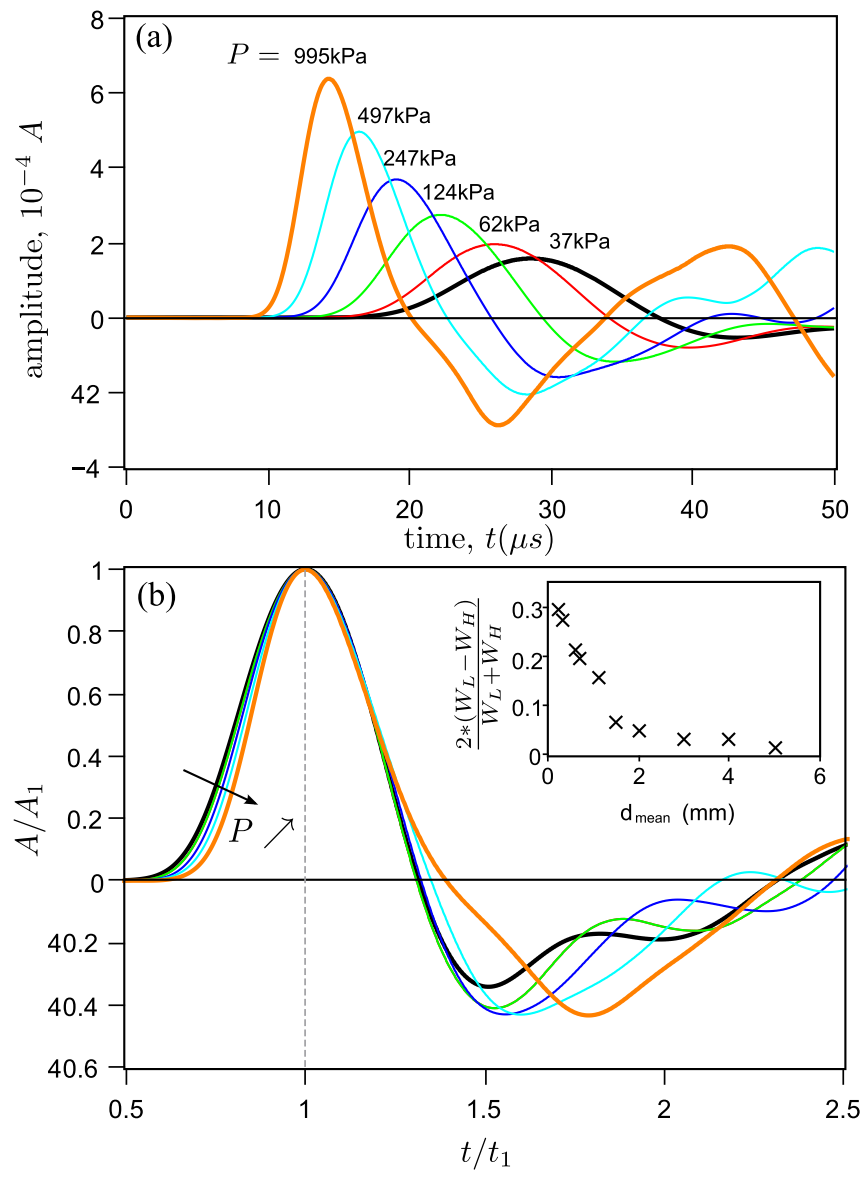

FIG. 4. (Color online) The effect of the confining pressure $P$ on the shape of the pulse signal measured at $L=11.5 \mathrm{~mm}$. (a) Decreasing the pressure leads to an increase of the pulse width. (b) Rescaling the time axis by $t_{1}$ and the amplitude axis by $A_{1}$ leads to collapse of pulse signals. Inset: Decreasing the pressure make increase the normalized width $W$ with $W_{H}=W(P=995 \mathrm{kPa})$ and $W_{L}=W(P=65 \mathrm{kPa})$.

showing that the viscoelastic dissipation does not significantly affect measurements of the normalized pulse width.

To verify whether the normalized width depends on the amount of disorder mentioned above, we investigate the evolution of the prefactor $C_{W}$ in granular packings with a mixture of glass and PMMA beads. A mixture of the beads of different nature is expected to increase the heterogeneity of stiffness and mass in the granular packings and accordingly the normalized width $W$. For a given volume fraction of glass beads $\phi_{\text {glass }}\left[=V_{\text {glass }} /\left(V_{\text {glass }}+V_{\text {pmma }}\right)\right]$, we determined the prefactor $C_{W}$ by fitting the normalized widths measured for three source-receiver distances. Figure 6 shows the evolution of prefactor $C_{W}$ as a function of the glass beads volume fraction $\phi_{\text {glass }}$. As expected, $C_{W}$ versus $\phi_{\text {glass }}$ presents a maximum. Instead, the elastic longitudinal modulus $C_{11}$ given by $C_{11}=$ $\rho_{m} V_{p}^{2}$ with $\rho_{m}$ the packing density of the granular mixture increases monotonically with the volume fraction of glass beads.

\section{Attenuation measurement by FFT}

To identify the origin of the pulse broadening, we investigate the wave attenuation and velocity dispersion in frequency 

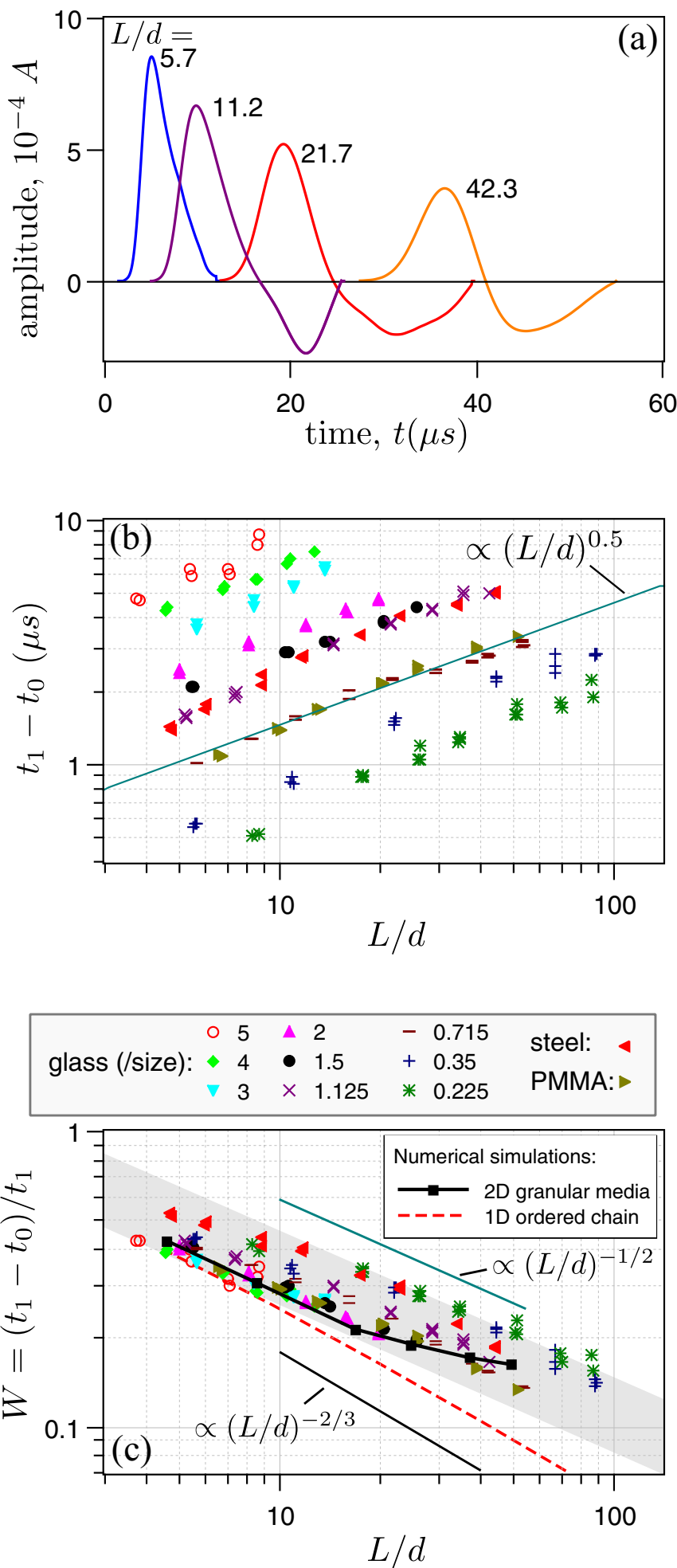

FIG. 5. (Color online) (a) Pulse broadening and decay through a glass bead packing of $d=0.715 \mathrm{~mm}$ under stress $P=995 \mathrm{kPa}$. The first period (plain line) of coherent wave front is used for the FFT analysis. (b) Width of the pulse measured as a function of the ratio $L / d$ for different granular media under $P=995 \mathrm{kPa}$. (c) Normalized pulse width $W=\left(t_{1}-t_{0}\right) / t_{1}$ plotted as a function of ratio $L / d$. Numerical results in 2D disordered granular packings [14] and $1 \mathrm{D}$ ordered chain simulation (dashed red line) are added here for comparison. The normalized width scales with $L$ as $W \sim C_{W}(L / d)^{-0.5}$.

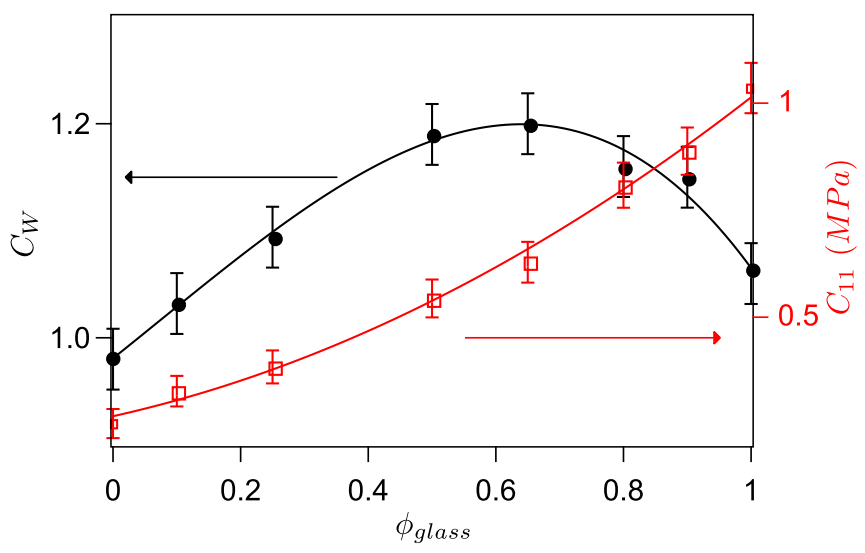

FIG. 6. (Color online) Pulse broadening due to disorder: the best fit values of prefactor $C_{W}$ and the elastic longitudinal modulus $C_{11}=$ $\rho_{m} V_{p}^{2}$ obtained as a function of glass beads volume fraction in a glass-PMMA beads mixture ( $d=0.615 \mathrm{~mm}, P=995 \mathrm{kPa})$.

domain. By a FFT analysis of coherent wave fronts measured at different source-receiver distances, it is possible to determine the wave attenuation $\alpha$ and velocity dispersion as a function of frequency $\omega$. As detailed elsewhere [25], the dispersion of the phase velocity is quite small in the range of frequency used here. Figures 7(a) and 7(b) show respectively the spectra associated with the pulses detected at different distances $L$ [Fig. 5(a)] and the attenuation $\alpha$ deduced from the ratio of spectra. For guidance, different scaling of attenuation on frequency $\left(\omega, \omega^{2}\right.$, and $\left.\omega^{4}\right)$ are recalled. We discuss later in $\mathrm{Sec}$. III C the possible mechanisms of wave attenuation.

\section{SIMULATION AND MODELING}

Despite the lack of theoretical models accurately describing the sound propagation and scattering in granular media, we seek to explain our main experimental observations by the simplified numerical simulations and the general models of elastic wave scattering in random media.

\section{A. Pulse broadening versus dispersion relationship}

We here consider two distinct mechanisms leading to the pulse broadening, i.e., wave attenuation $\alpha(\omega) \equiv k^{\prime \prime}(\omega)$ and velocity dispersion $V(\omega) \equiv \omega / k^{\prime}(\omega)$ where $k(\omega)=k^{\prime}(\omega)+$ $i k^{\prime \prime}(\omega)$ is the complex wave number. To this end, we investigate the propagation of a plane wave $a(x, t)$ along $x$, related to its inverse Fourier transform $\tilde{a}$ by

$$
a(x, t)=\frac{1}{2 \pi} \int_{-\infty}^{+\infty} e^{-i \omega t} A(\omega) e^{i k(\omega) x} d \omega
$$

with $\tilde{a}=A(\omega) e^{i k(\omega) x}$. For a Dirac-like pulse propagation, one has $A(\omega)=1$. Two properties of the Fourier transform are recalled which are useful for the following discussions. (i) Translation: if $g(t)=f\left(t-t_{1}\right)$, then $\widetilde{g}(\omega)=e^{i \omega t_{1}} \widetilde{f}(\omega)$. (ii) Scaling: if $g(t)=f(a t)$, then $\widetilde{g}(\omega)=\left|\frac{1}{a}\right| \widetilde{f}\left(\frac{\omega}{a}\right)$.

We first study the effect of the velocity dispersion $V(\omega)=$ $\omega / k^{\prime}(\omega)$ on the pulse broadening in a 1D mass-spring chain composed of identical spheres of radius $R$. There is no attenu- 

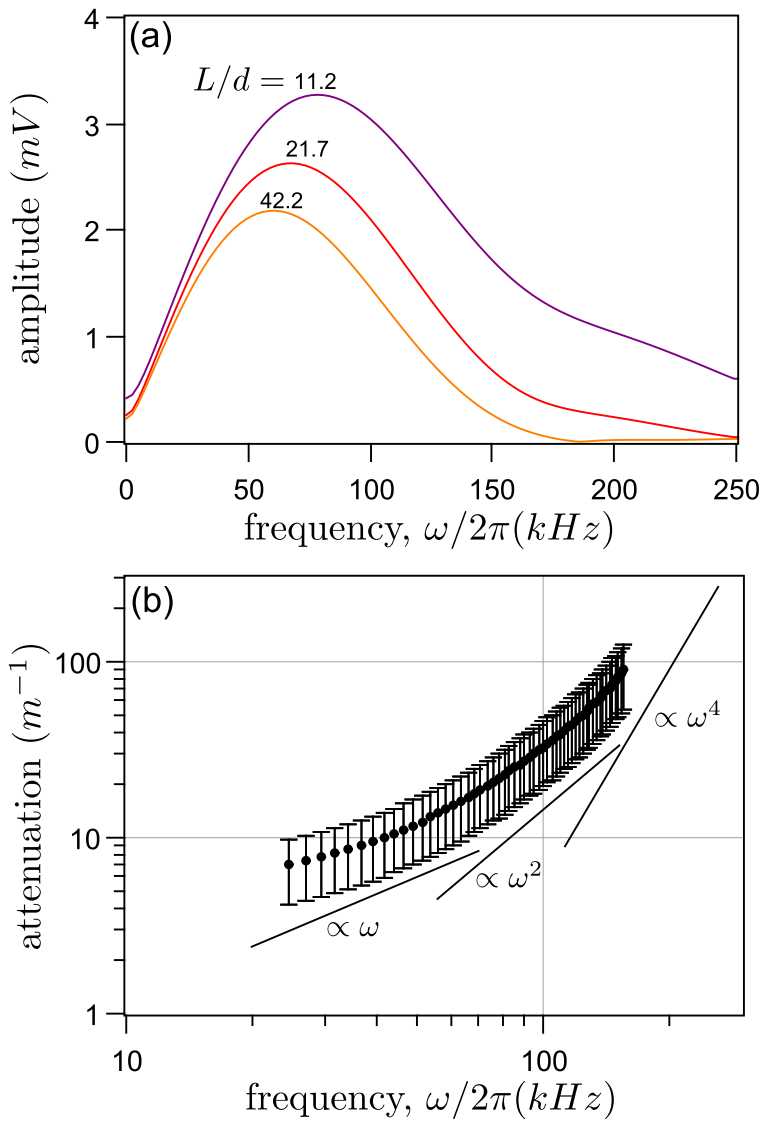

FIG. 7. (Color online) (a) Spectra associated with the pulses detected at different distances $L$ [Fig. 5(a)]. (b) Attenuation $\alpha$ as a function of the pulsation $\omega$ in $d=0.715 \mathrm{~mm}$ glass beads $(P=$ $995 \mathrm{kPa}$ ). Error bars correspond to measurements using different ratios of spectra, and different scaling laws are added for guidance.

ation in such a system $\left(k^{\prime \prime}=0\right)$ and the dispersion relationship is given by $k=(1 / R) \sin ^{-1}\left(\frac{\omega R}{V}\right)$ with $V$ the longitudinal wave velocity in the long wavelength limit. If the wavelength is long enough, the wave number can be approximated by $k=\frac{\omega}{c}+\frac{1}{6}\left(\frac{\omega}{c}\right)^{3} R^{2}$ [14], and the Fourier transform of the signal will be given by $e^{i\left((\omega / V) x+(1 / 6)(\omega / V)^{3} R^{2} x\right)}=e^{i \omega t_{1}} e^{i\left((1 / 3)\left(\omega / \omega_{1}\right)^{3}\right)}$ with $t_{1}=\frac{x}{V}$ and $\omega_{1}=\left(\frac{2 V^{3}}{R^{2} x}\right)^{1 / 3}$. The first term $e^{i \omega t_{1}}$ can be Fourier transformed using the translation property, and the second one $e^{i\left((1 / 3)\left(\omega / \omega_{1}\right)^{3}\right)}$ corresponds to the Fourier transform of the Airy function $\operatorname{Ai}(-x)$ scaled by the pulsation $\omega_{1}$. As shown in [14], the resultant temporal signal can be written as $a(x, t)=\omega_{1} \operatorname{Ai}\left[\omega_{1}\left(t_{1}-t\right)\right]=\omega_{1} \operatorname{Ai}\left\{\omega t_{1}\left[\left(t_{1}-t\right) / t_{1}\right]\right\}$. With the rescaled time axis $\left(t_{1}-t\right) / t_{1}$, the normalized width of the signal is then given by $W \sim\left(\omega_{1} t_{1}\right)^{-1}=\left(\frac{R^{2}}{2 x^{2}}\right)^{1 / 3} \propto x^{-2 / 3}$, namely,

$$
W \propto L^{-2 / 3}
$$

which implies a pulse broadening $\left(t_{1}-t_{0}\right) \sim W t_{1} \sim L^{1 / 3}$, as previously found [14].

Second, we examine a pulse propagation in a $1 \mathrm{D}$ random medium where the phase velocity $V=\omega / k^{\prime}$ is constant, dispersionless. However, there is an attenuation given by $\alpha=k^{\prime \prime}(\omega)=\left[\sigma_{K}^{2} L_{c}^{n-1}\right]\left(\frac{\omega}{V}\right)^{n}$ with $n$ an integer; the underlying physics (and the parameters in the bracket) of such a system is detailed in Sec. III C below. The Fourier transform of the temporal signal is then $\tilde{a}(\omega)=e^{i k^{\prime} x} e^{-k^{\prime \prime} x}=e^{i \omega t_{1}} e^{-\left(\omega / \omega_{1}\right)^{n}}$ with $t_{1}=\frac{x}{V}$ and $\omega_{1}=\left(\frac{V^{n}}{\sigma_{K}^{2} L_{c}^{n-1} x}\right)^{1 / n}$. By considering the properties of the Fourier transform, the temporal signal is $a(x, t)=$ $\omega_{1} s\left[\omega_{1}\left(t-t_{1}\right)\right]$ where the function $s$ is the inverse Fourier transform of $e^{-\omega^{n}}$. With the rescaled time axis $\left(t_{1}-t\right) / t_{1}$, the normalized width of the signal is then $W \sim\left(\omega_{1} t_{1}\right)^{-1}=$ $\left(\sigma_{K}\right)^{2 / n}\left(\frac{L_{c}}{x}\right)^{(n-1) / n}$. In 1D randomly layered media, one has $n=2$ (see details below in Sec. III C) leading to $\alpha \sim \omega^{2}$ and $W$ at $(x=L)$

$$
W \propto L^{-1 / 2}
$$

\section{B. Simulations in 1D chains of elastic lattices}

The use of normalized variables allows us to compare our experimental results to numerical simulations. As shown in Fig. 5(c), the numerical results obtained in disordered 2D granular media [14] compare quite well with our experimental results. Moreover, the velocity dispersion in 1D ordered chains leads to a power law scaling of $W$ on distance as $(L / d)^{-2 / 3}$ [Eq. (3)] as found in [14]. However, the normalized widths obtained in disordered packing scales with distance as $(L / d)^{-1 / 2}$ [similar to Eq. (4)] and have also values larger than those deduced from ordered packings.

In order to evaluate the effect of scattering attenuation on the pulse broadening, we perform the simulation in a 1D disordered mass-spring chain in the presence of the correlation length similar to random media by Fouque et al. [19]. More specifically, we consider a $1 \mathrm{D}$ chain of mass spring consisted of subchains of length $L_{i}$ and stiffness $D_{i}$ which is uniform inside each subchain [Fig. 8(a)]. Assuming an exponential distribution of the subchain length $L_{i}$,

$$
p\left(L_{i}\right)=\left(1 / L_{0}\right) e^{-L_{i} / L_{0}},
$$

we then obtain a mean length of the subchains equal to $L_{0}$. If the inverse of stiffness is given by $D^{-1}=\bar{D}^{-1}\left(1+v_{D}\right)$ with $\bar{D}^{-1}$ the mean value and $v_{D}$ the fluctuation uniformly distributed in the range $\left[-v_{\max },+v_{\max }\right]$, we may readily verify $\left\langle v_{D}\right\rangle=0$ and the variance $\left\langle v_{D}^{2}\right\rangle=\sigma_{D}^{2}=\left(v_{\max }\right)^{2} / 3$. To determine the correlation length associated with such a disordered chain, we compute the correlation function of the stiffness fluctuation $\left\langle v_{D}(x) v_{D}(x+\Delta x)\right\rangle$ via ensemble average between two points separated by a distance $\Delta x$. Figure 8(c) shows the normalized correlation function (by $\sigma_{D}^{2}$ ) versus $\Delta x$, calculated for the different $L_{0}$. If we defined the correlation length $L_{c}$ corresponding to a decrease of the normalized correlation to about $0.4\left(\approx e^{-1}\right)$, we deduce a correlation length $L_{c} \approx L_{0}$ from Fig. 8(c).

Simulations of the pulse propagation are performing by solving the eigenproblem of the linear spring system at given initial conditions. The equation of motion of the $N$ beads is given by [Fig. 8(b)]

$$
m \ddot{u}_{j}=D_{j+1}\left(u_{j+1}-u_{j}\right)+D_{j}\left(u_{j-1}-u_{j}\right) .
$$

This equation can be expressed in a matrix form $m \ddot{\mathbf{u}}=\mathbf{D} \cdot \mathbf{u}$ where $\mathbf{u}$ is the displacement vector of each bead (only the translational motion is considered here), $m$ is the constant mass of beads, and $\mathbf{D}$ is a $N \times N$ matrix (for simplicity $m$ and $\bar{D}$ are here set to 1). Eigenfrenquencies $\omega_{n}$ of this linear 


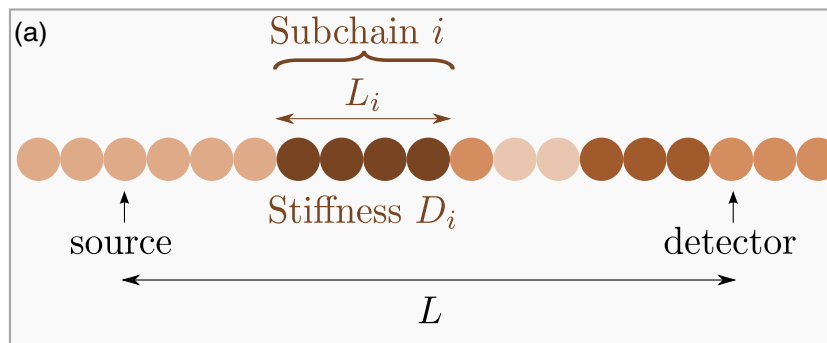

(b)
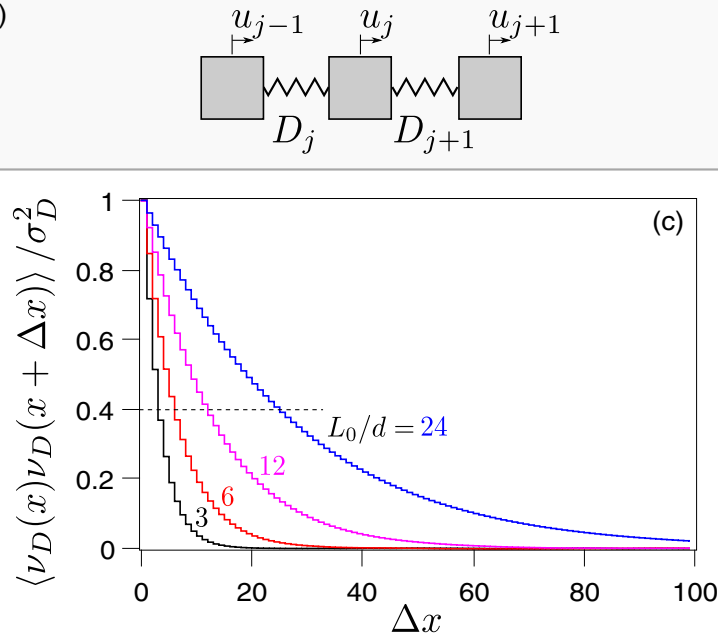

FIG. 8. (Color online) (a) Random 1D chain (see text). (b) The interaction between the nearest neighboring beads. (c) Autocorrelation function of the fluctuation $v_{D}$ of the inverse stiffness $D^{-1}$ vs separate distance $\Delta x$ for different mean lengths $L_{0}$.

system are the square roots of the eigenvalues of the matrix $\mathbf{D} / m$. The oscillations of beads are given by the superposition of the eigenmodes: $\mathbf{u}(t)=\sum_{n} A_{n} u_{n} \cos \left(\omega_{n} t\right)$ where $u_{n}$ are the eigenvectors of the matrix $\mathbf{D} / m$ and the amplitudes $A_{n}$ are determined by the initial conditions. To prevent the wave reflections at the edges, the source and the detector are placed in the middle of the chain. Assuming that at $t=0$ the particle displacement $u$ located at the source is equal to 1 , the propagation of the pulse for various source-detector distances $L$ is then computed with the displacement $u(t)$, averaged over 100000 configurations.

Figure 9(a) shows the evolution of the coherent wave front where the shape of the wave front evolves during the propagation and tends to a Gaussian-like pulse at long distance. The normalized width of the pulse is plotted in Fig. 9(b) as a function of the source-detector distance for the case $\sigma_{D}=0.29$. The variance $\sigma_{D}$ slightly increases the normalized width and the variation of the mean or correlation length further enhances the effect on $W$. Figure 9(c) depicts the pulsed wave attenuation observed in frequency domain. It scales as $\omega^{2}$ and increases with the mean length of subchains $L_{0}$.

\section{Models of elastic wave scattering and dissipation}

Finally, we compare our experimental and numerical results with the theoretical models of elastic wave propagation in random or granular media. In these scattering media, the models seek to relate the attenuation of coherent waves to the statistical properties of random media such as the
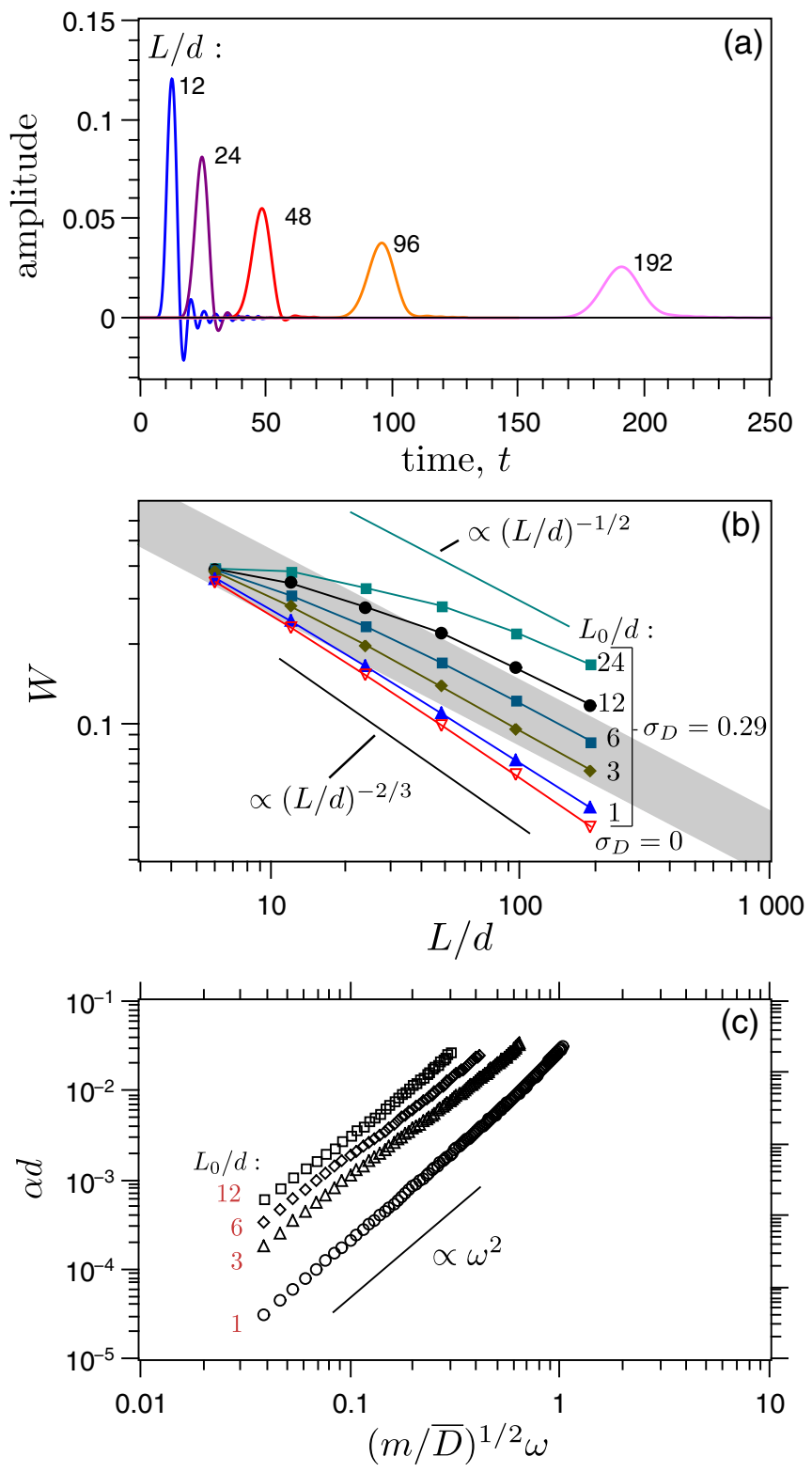

FIG. 9. (Color online) (a) Pulse broadening and decay of the coherent compressional wave propagating through a $1 \mathrm{D}$ chain $\left(\sigma_{D}=\right.$ 0.29 and $L_{0} / d=6$ ). (b) Normalized signal width as a function of $L / d$. In disordered chains, the signal is averaged on 100000 configurations. The gray band corresponds to the experimental data dispersion [Fig. 5(c) is added]. (c) Attenuation (over a distance of $d$ ) of the coherent wave propagating through a $1 \mathrm{D}$ chain for $\sigma_{D}=0.29$ and various correlation lengths.

elastic modulus $K$ and the density $\rho[18,19,26]$, for instance introducing a characteristic length $L_{c}$ of the fluctuation $v_{K}=$ $\Delta K^{-1} / \bar{K}^{-1}$ (see below).

Consider 1D random layers with the similar statistical properties as those investigated above in 1D disordered chains and free of dispersion and dissipation. Assuming $L_{c} \ll \lambda \ll L$ and a high level of disorder corresponding to a variance $\left\langle v_{K}^{2}\right\rangle=\sigma_{K}^{2} \sim 1$, Fouque et al. [19] show that the wave form of a coherent pulse tends to a Gaussian signal at long distance of propagation [Fig. 9(a)]; it propagates at an effective velocity 


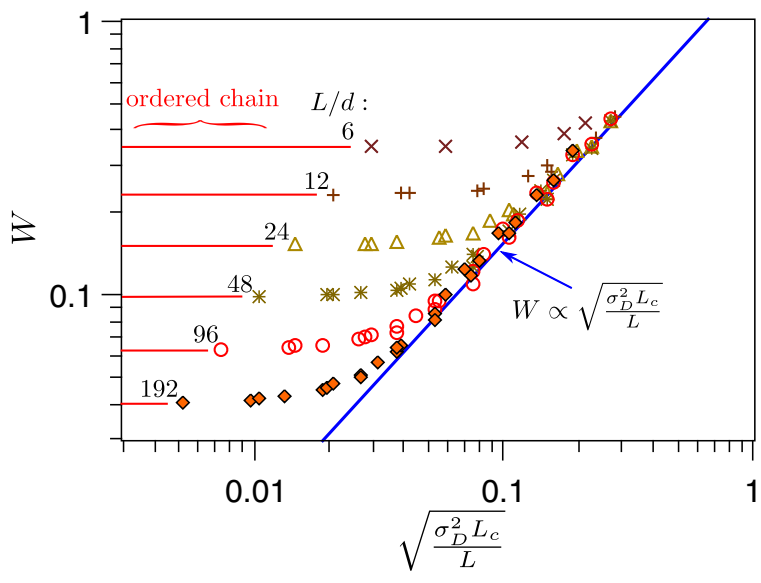

FIG. 10. (Color online) Normalized width $W$ as a function of $\sqrt{\sigma_{D}^{2} L_{c} / L}$ for various source-detector distance $L / d$. Only cases satisfying $L>L_{c}$ are shown. For high disorder, the pulse width $W$ tends to $\sqrt{\ln (10)} \sqrt{\sigma_{D}^{2} L_{c} / L}$ predicted by Eq. (7).

$$
\begin{aligned}
\bar{V}=\sqrt{\bar{K} / \bar{\rho}}: & \\
a(L, t) & =\frac{1}{\sqrt{2 \pi w_{L}^{2}}} e^{-(t-L / \bar{V})^{2} / 2 w_{L}^{2}},
\end{aligned}
$$

where $w_{L}=\left(\gamma L / 2 \bar{V}^{2}\right)^{1 / 2}$ governs the pulse width and $\gamma=$ $\int_{0}^{\infty}\left\langle v_{K}(0) v_{K}(x)\right\rangle d x=\sigma_{K}^{2} L_{c}$ is determined by the product of the variance $\sigma_{K}^{2}$ and the correlation length $L_{c}$. Equation (7) implies that the normalized width $W \sim w_{L} /(L / \bar{V})$ scales with propagation distance $L$ as $\left(\sigma_{K}^{2} L_{c} / L\right)^{1 / 2}$, and that the attenuation of the pulse is given by $\alpha=\gamma\left(\frac{\omega}{2 \bar{V}}\right)^{2}$.

Figure 10 depicts numerical results from simulations in disordered chains, regarding the normalized width $W$ for various source-detector distances $L / d$ as a function of a combined parameter $\left(\sigma_{D}^{2} L_{c} / L\right)^{1 / 2}$. More specifically, at a given distance $L$, we determine $W$ by varying the variance $\sigma_{D}$ or/and the correlation length $L_{c}$. The stack data show that for high disorder $W$ scales as $\left(\sigma_{D}^{2} L_{c} / L\right)^{1 / 2}$ as predicted above, while for the weak level of disorder, i.e., $\sigma_{D}^{2}\left(\frac{L_{c}}{d}\right) \lesssim\left(\frac{L}{d}\right)^{-1 / 3}$, $W$ tends to the constant values as those determined in ordered 1D elastic chains (represented by the horizontal solid lines). In terms of the dispersion relationship for the high level of disorder, the attenuation is given by $\alpha=\sigma_{D}^{2} L_{c} k^{2} / 4 \sim \omega^{2}$, consistent with the results shown in Fig. 9(c).

In 3D random media without dispersion and dissipation $[18,26]$, the attenuation may be expressed in the limit case $k^{2} \gamma L \ll 1$, by

$$
\alpha \sim \sigma_{K}^{2} L_{c}^{n-1} k^{n} \sim \omega^{n},
$$

where $n=2$ corresponds to the large scale fluctuation $\left(L_{c} \gg\right.$ $\lambda$ ) with a strongly anisotropic scattering, identical to the above result obtained in 1D random media [see also Fig. 9(c)], and $n=4$ corresponds to the small scale fluctuation $\left(L_{c} \ll \lambda\right)$ with an isotropic Rayleigh-like scattering [26].

Regarding the experimental data of attenuation shown in Fig. 7 and in [27], these two mechanisms of scattering invoked here corresponding to $\alpha \sim \omega^{2}$ and $\alpha \sim \omega^{4}$, respectively, may explain qualitatively the measurements at the high-frequency range (note that the former appears more significant in the present work), due to the heterogeneous and anisotropic structure of the contact force networks. However, at the low-frequency range, we experimentally observe a different behavior of attenuation $\alpha \sim \omega^{1}$. As mentioned before, the viscoelastic dissipation related to the particle material seems to be negligible here. Nevertheless, the wave dissipation around the bead contacts via thermoelastic relaxation might be a mechanism responsible for such kind of attenuation, as proposed by Wang and Santamarina [28], when the characteristic time is comparable with $\sim 1 / \omega$.

\section{CONCLUSION}

We have studied the pulse broadening of coherent sound waves propagating in granular media. The evolution of the pulse width of the coherent compressional wave is analyzed in terms of the dispersion relationship, including wave attenuation and velocity dispersion. The pulse broadening measured in various disordered bead packings reveals both a different scaling law on distance of propagation $L$ and larger values, compared to analytical and numerical results only invoking the dispersion effect as that found in ordered elastic lattices. The numerical simulations carried out in 1D disordered elastic systems composed of subchains show that the pulse broadening may be caused by scattering attenuation. More specifically, the normalized pulse width $W$ by the wave propagation time scales with distance as $\propto L^{-1 / 2}$ instead $\propto L^{-2 / 3}$ due to the dispersion, and significantly depends on the product of the inverse stiffness variance $\sigma_{D}^{2}$ and the correlation length $L_{c}$. Our experimental and numerical results are consistent with theoretical models of elastic wave scattering in $1 \mathrm{D}$ and $3 \mathrm{D}$ random continuous media, suggesting a dominant attenuation mechanism $\alpha \sim \omega^{2}$ possibly due to an anisotropiclike scattering. Further works are still necessary to highlight the underlying physics of attenuation at the low-frequency range.

For future studies, the effects of disorder via mass or spring fluctuations found by numerical simulation in $1 \mathrm{D}$ chains need to be investigated in $2 \mathrm{D}$ or $3 \mathrm{D}$ disordered elastic networks. In these cases, the additional topological disorder appears and the absence of a reference medium prevents actually the analytical model of sound propagation [5]. Numerical simulation may help us to better understand the elastic wave propagating in granular media.
[1] H. M. Jaeger, S. R. Nagel, and R. P. Behringer, Rev. Mod. Phys. 68, 1259 (1996).

[2] X. Jia, C. Caroli, and B. Velicky, Phys. Rev. Lett. 82, 1863 (1999).
[3] X. Jia, J. Laurent, Y. Khidas, and V. Langlois, Chin. Sci. Bull. 54, 4327 (2009).

[4] P. J. Digby, J. Appl. Mech. 48, 803 (1981).

[5] B. Velický and C. Caroli, Phys. Rev. E 65, 021307 (2002). 
[6] H. A. Makse, N. Gland, D. L. Johnson, and L. Schwartz, Phys Rev. E 70, 061302 (2004).

[7] S. Wildenberg, M. van Hecke, and X. Jia, Europhys. Lett. 101, 14004 (2013).

[8] M. Wyart, L. E. Silbert, S. R. Nagel, and T. A. Witten, Phys. Rev. E 72, 051306 (2005).

[9] C. Song, P. Wang, and H. A. Makse, Nature (London) 453, 629 (2008).

[10] I. Agnolin, J.-N. Roux, P. Maassad, X. Jia, and P. Mills, in Powders \& Grains 2005, edited by R. García Rojo, H. Herrmann, and S. McNamara (Balkema, Rotterdam, 2005), p. 313.

[11] M. van Hecke, J. Phys.: Condens. Matter 22, 033101 (2010).

[12] M. Arroyo, D. Muir Wood, and P. D. Greening, Géotechnique 53, 337 (2003).

[13] E. T. Owens and K. E. Daniels, Soft Matter 9, 1214 (2013).

[14] E. Somfai, J. N. Roux, J. H. Snoeijer, M. van Hecke, and W. van Saarloos, Phys. Rev. E 72, 021301 (2005).

[15] O. Mouraille, W. A. Mulder, and S. Luding, J. Stat. Mech. (2006) P07023.

[16] J. O’Donovan, C. O'Sullivan, and G. Marketos, Granular Matter 14, 733 (2012).

[17] G. S. Grest, S. R. Nagel, and A. Rahman, Phys. Rev. Lett. 49, 1271 (1982).
[18] S. M. Rytov, Y. A. Kravtsov, and V. I. Tatarski, Elements of Random Fields, Principles of Statistical Radiophysics No. 4 (Springer-Verlag, Berlin, 1989).

[19] J.-P. Fouque, J. Garnier, G. Papanicolaou, and K. Solna, Wave Propagation and Time Reversal in Randomly Layered Media (Springer, New York, 2007), Chap. 8.2.3.

[20] J. H. Page, H. P. Schriemer, A. E. Bailey, and D. A. Weitz, Phys. Rev. E 52, 3106 (1995).

[21] X. Jia, Phys. Rev. Lett. 93, 154303 (2004).

[22] J. P. Weight, J. Acoust. Soc. Am. 81, 815 (1987).

[23] B. Audoin and J. Roux, Ultrasonics 34, 25 (1996).

[24] H. A. Makse, D. L. Johnson, and L. M. Schwartz, Phys. Rev. Lett. 84, 4160 (2000).

[25] Y. Yang, Ph.D. thesis, Université Paris-Est Marne-la-Vallée, 2013 (in French).

[26] L. A. Chernov, Wave Propagation in a Random Medium (McGraw-Hill, New York, 1960).

[27] T. Brunet, X. Jia, and P. Johnson, Geophys. Res. Lett. 35, 19308 (2008).

[28] Y.-H. Wang and J. C. Santamarina, Granular Matter 9, 365 (2007). 